\title{
Quantitative analysis of lymph nodes in neck dissection specimens. Morphologic study ${ }^{1}$
}

Fábio de Aquino Capelli', Vitor Ribeiro Paes"I, Mariangela Marinheiro Machado III, Camila Lohmann Menezes ${ }^{\mathrm{III}}$, Pablo Rodrigo Andrade da Silva ${ }^{I I I}$, Sheila Aparecida Coelho Siqueira ${ }^{\mathrm{IV}}$, Venâncio Avancini Ferreira Alvesv, Leandro Luongo Matos"II, Claudio Roberto Cernea $^{\text {III }}$

DOI: http://dx.doi.org/10.1590/S0102-865020160070000001

IMD, Department of Head and Neck Surgery, School of Medicine, Universidade de São Paulo (USP), Sao Paulo-SP, Brazil. Analysis of data, manuscript writing, design and supervise all phases of the study.

IIMD, Department of Pathology, School of Medicine, USP, Sao Paulo-SP, Brazil. Analysis of data, histopathological examinations, design and supervise all phases of the study.

III Graduate student, School of Medicine, USP, Sao Paulo-SP, Brazil. Acquisition of data.

${ }^{\text {IV }} \mathrm{PhD}$, Department of Pathology, School of Medicine, USP, Sao Paulo-SP, Brazil. Design and supervise all phases of the study.

${ }^{v} \mathrm{PhD}$, Chairman, Full Professor, Department of Pathology, School of Medicine, USP, Sao Paulo-SP, Brazil. Design and supervise all phases of the study.

${ }^{V}$ IPhD, Assistant Professor, Department of Head and Neck Surgery, School of Medicine, USP (LIM 28) and Instituto do Câncer do Estado de São Paulo (ICESP), Brazil. Analysis of data, manuscript writing, critical revision, design and supervise all phases of the study.

${ }^{V I I} \mathrm{PhD}$, Associate Professor, Head Division, Department of Head and Neck Surgery, School of Medicine, USP, Sao Paulo-SP, Brazil. Critical revision, design and supervise all phases of the study.

\section{ABSTRACT}

PURPOSE: To quantify the amount of lymph nodes harvested in modified radical neck dissection.

METHODS: Cross-sectional anatomical study conducted in 28 non-preserved cadavers.

RESULTS: The mean number of lymph nodes found in each nodal level of the 56 modified radical neck dissections performed were: level IA - 1.5 (95\% CI: 1.1 - 1.8), level IB - 2.5 (95\% CI: 2.1 - 2.9), level IIA - 7.2 (95\% CI: 6.0 - 8.5), IIB level - 6.5 (95\% CI: 5.5 - 7.4), level III - 6.6 (95\% CI: 5.7 - 7.4), level IV - 8.6 (95\% CI: 7.1 - 10.1), level V - 11 (95\% CI: 9.2 - 12.7), totalizing 43.8 lymph nodes (95\% CI: $40.3-47.4)$.

CONCLUSION: The results defined a parameter in relation to the minimum recommended nodal yield in a modified radical neck dissection, as well as the number of lymph nodes in each level of this dissection, performed in clinical practice.

Key words: Lymph Node Excision. Neck Dissection. Head and Neck Neoplasms. Anatomy. Cadaver. 


\section{Introduction}

Head and neck cancers have great potential for regional spread by metastasis to lymph nodes (LNs). Except for the presence of distant metastases, the most adverse prognostic factor in head and neck squamous cell carcinoma (HNSCC) is regional nodal involvement ${ }^{1}$. Treatment of this disease requires the knowledge of this dissemination mechanism, the distribution of LNs in specific anatomical regions and appropriate surgical techniques for the approach, which is called neck dissection (ND).

The number of LNs expected to be retrieved in a specimen of modified radical neck dissection (MRND) is uncertain $^{2}$, with variable references in the literature (mean of 31$55 \mathrm{LNs}$ ), depending on the processing method by the pathologist and the surgeon's ability for the complete removal of these nodes located within the anatomic boundaries of the neck nodal levels ${ }^{3-9}$. Pathological assessment (macroscopic and microscopic) of the ND specimen becomes extremely important, providing data as the total number of LN recovered (whether metastatic or not), the size of the metastasis, the presence of extracapsular spread or micrometastasis. Pathological status of the neck ( $\mathrm{pN}$ at TNM classification) is one of the determining factors in the indication of adjuvant therapy ${ }^{6}$.

This study aims to quantify the number of lymph nodes retrieved in modified radical neck dissection specimens performed in a series of non-preserved cadavers, and to verify if there is any relation between the number of lymph nodes and demographic and anthropometric data of the individuals.

\section{Methods}

The authors declare no conflict of interest and all the cadavers were treated with the highest respect. The research protocol was approved by the Institutional's Research Ethics Committees (protocol number: 312/2013).

Cross-sectional anatomical study conducted in 28 nonpreserved cadavers.

\section{Sample estimation}

Mean estimation modelling was chosen for sample size calculation $^{10}$, and it was based on the study of Agrama et al. ${ }^{3}$ that summarized the results of six studies, totalizing 5065 valid patients and establishing as weighted mean 35.2 LNs in a radical ND and weighted standard deviation of 5.9 LNs. Thus, using this study as reference and adopting standard deviation of 6 LNs and maximum error of the estimative of $1.75 \mathrm{LNs}$, with an overall significance level of 0.05 , the sample size calculated was 45 ND. Estimating a possible loss of data of $25 \%$, the final number of 56 specimens $(28$ cadavers) was assumed to be the goal.

\section{Anatomic study}

The cadavers were placed in supine position with the head in neutral position and arms parallel to the body. A subscapular cushion was used for neck extension. Demographic (sex, age and race) and anthropometric (weight, height and body mass index BMI) data were recorded.

Exclusion criteria were: previous cervical manipulation, the presence of head and neck neoplasm and any anatomic abnormality on the neck.

A wide cervicothoracic incision was performed on each cadaver and the steps of modified radical neck dissection were reproduced, with the removal of fibrofatty tissue containing the deep cervical lymph nodes, in a level-by-level basis, according to the conventional classification and anatomical limits ${ }^{11}$ (Figure 1). As a small change in the technique, the sternocleidomastoid muscle was sectioned in its upper third (on the junction level with the spinal accessory nerve) to provide full access to the jugular LNs. The final aspect of the dissection and the retrieved bilateral cervical nodal levels are shown in Figure 2.
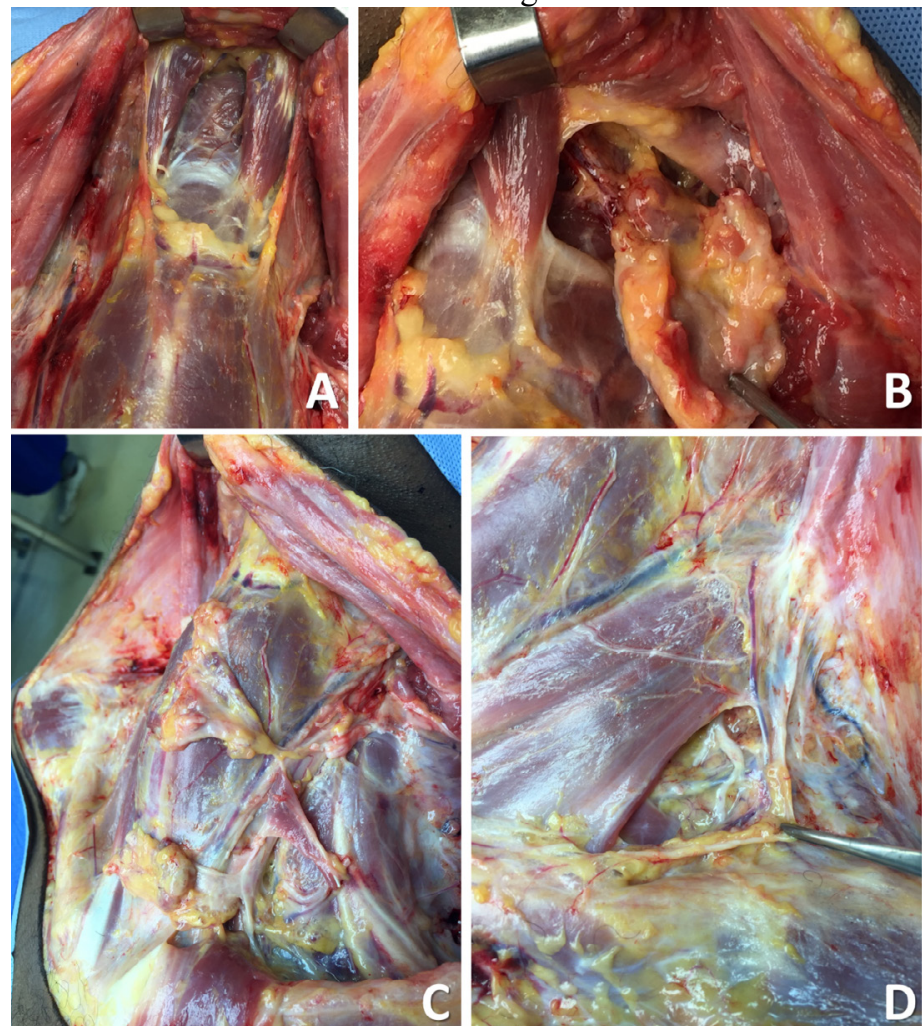

FIGURE 1 - Radical modified neck dissection performed in the left side neck of a non-preserved cadaver; (A) Leve IA (within the two anterior bellies of the digastric muscles) after the specimen removed; (B) Level IB (within the two bellies of the digastric muscle and the mandible) with the submandibular gland and lymph nodes retracted; (C) Levels II. III and IV (jugular chain of lymph nodes) with the specimen removed and the sternocleidomastoid muscle folded; (D) Level V (posterior triangle) showing the spinal accessory nerve. 

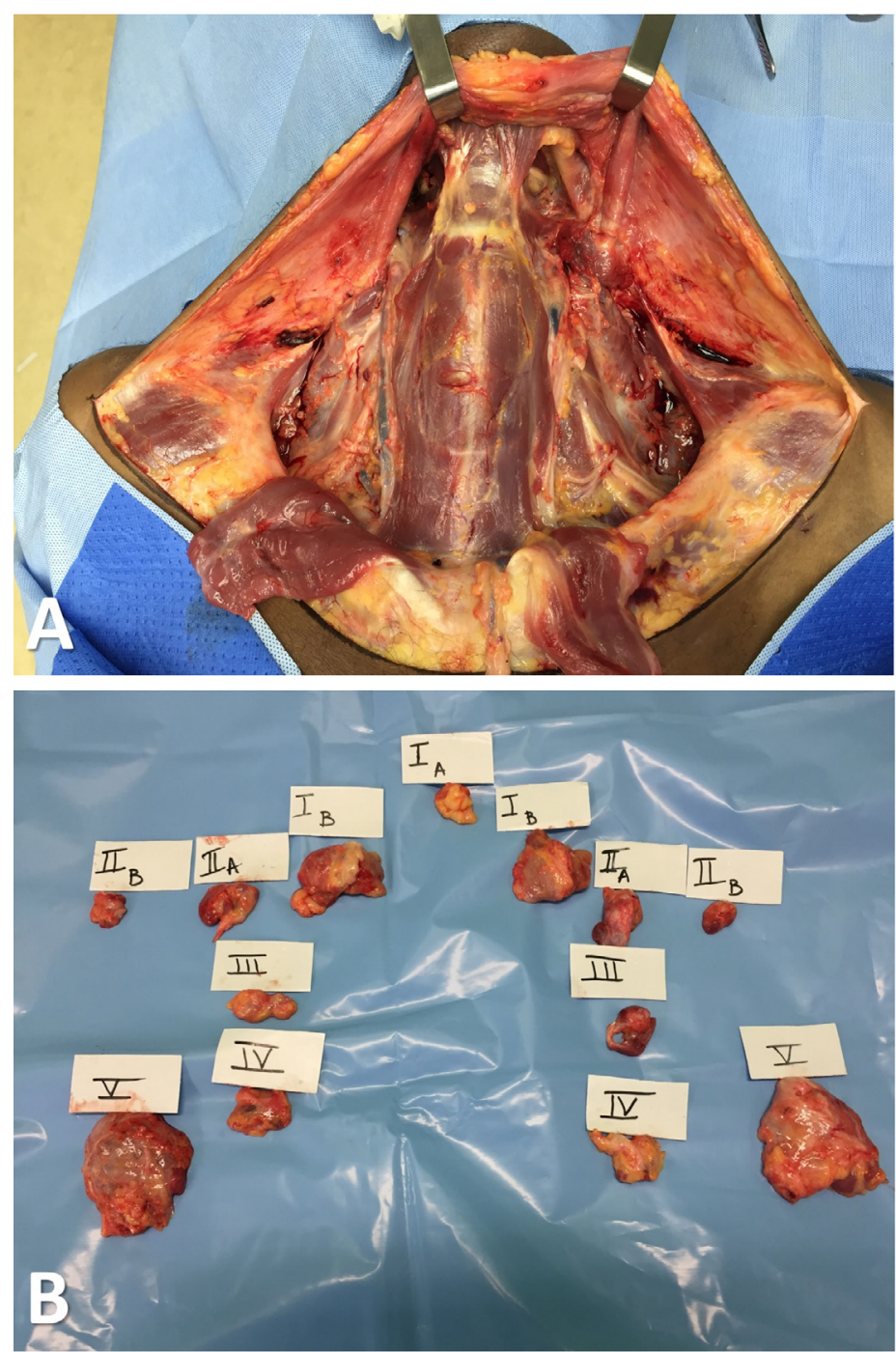

FIGURE 2 - Final aspect of the dissection (A) and the nodal levels of both neck sides removed (B).

The harvested tissue of each level and sublevel, dissected within the limits recommended by the Committee for Neck Dissection Classification of the American Head and Neck Society (AHNS) ${ }^{12}$, was delivered to the pathologist and dissected manually. Palpable LNs were placed in cassettes and separately preserved in $10 \%$ formalin before pathological examination. The remaining tissue was sectioned into 3-5 $\mathrm{mm}$ pieces and paraffin embedded. Paraffin block was cut in $4 \mu \mathrm{m}$ histological sections and stained with hematoxylin and eosin for further evaluation of microscopic LNs.

Microscopic analysis were accessed by the same pathologist for histological confirmation of the manually dissected LNs. To be considered a LN, microscopically the structure should be described as an encapsulated lymphoid tissue aggregate with a marginal sinus ${ }^{2,11}$.

\section{Statistical analysis}

The values of the qualitative variables were described using absolute and relative frequencies. The distribution of the discrete quantitative variables was defined as non-parametric according to Kolmogorov-Smirnov test. These variables were described by mean and standard deviation and also by range, median, interquartile range and $95 \%$ confidence interval. MannWhitney test was performed for the comparison of quantitative data between groups. Correlation between quantitative variables was performed using Spearman's correlation test. All statistical analysis were performed using SPSS $^{\circledR}$ version 17.0 software (SPSS ${ }^{\circledast}$ Inc; Illinois, USA) and a significance level of $5 \%$ was adopted $(\mathrm{P} \leq 0.05)$.

\section{Results}

The sample consisted of 28 cadavers (56 specimens of modified radical neck dissection) and 2381 LNs were analyzed. Eighteen (64.3\%) were male and the mean age was $67.4 \pm 10.3$ years. Fifteen subjects were classified as Caucasian (53.6\%), 12 Afro-descendants (42.9\%) and one Asian (3.6\%), with mean weight of $59.6 \pm 13.3 \mathrm{~kg}$, mean height of $164 \pm 9.1 \mathrm{~cm}$ and mean body mass index (BMI) of $22.0 \pm 3.9 \mathrm{~kg} / \mathrm{m}^{2}$.

The analysis of the nodal yield in the right side of the neck showed a mean number of $44.4 \mathrm{LNs}$ (range: 26 - $99 \mathrm{LNs}$ ) and level V had the highest mean of LNs (11.9 LNs) compared to the other nodal levels (Table 1).

TABLE 1 - Descriptive of the right side of the neck.

\begin{tabular}{ccccccc}
\hline N=28 & Mean & Median & CI (95\%) & SD & $\begin{array}{c}\text { Mini- } \\
\text { mum }\end{array}$ & $\begin{array}{c}\text { Maxi- } \\
\text { mum }\end{array}$ \\
\hline IA & 1.5 & 1 & $1.0-1.9$ & 1.2 & 0 & 4 \\
IBD & 2.5 & 2 & $2.0-3.1$ & 1.5 & 1 & 8 \\
IIAD & 8 & 6 & $5.7-10.2$ & 5.8 & 1 & 24 \\
IIBD & 6.3 & 5.5 & $4.7-8.0$ & 4.2 & 0 & 15 \\
IIID & 7.1 & 7 & $6.0-8.2$ & 2.9 & 2 & 12 \\
IVD & 7.0 & 7 & $5.1-8.9$ & 4.9 & 0 & 23 \\
VD & 11.9 & 10 & $9.1-14.8$ & 7.4 & 1 & 34 \\
Total D & 44.4 & 40 & $38.2-50.6$ & 16 & 26 & 99 \\
\hline
\end{tabular}

CI. Confidence interval; SD. Standard deviation

On the left side, the mean number of retrieved LNs was 43.3 (range: $19-64 \mathrm{LNs}$ ), with level IV and V showing the highest mean LNs (10.2 and 10 LNs respectively) in comparison to other levels (Table 2). 
TABLE 2 - Descriptive of the left side of the neck.

\begin{tabular}{ccccccc}
\hline $\mathrm{N}=28$ & Mean & Median & CI (95\%) & SD & $\begin{array}{c}\text { Mini- } \\
\text { mum }\end{array}$ & $\begin{array}{c}\text { Maxi- } \\
\text { mum }\end{array}$ \\
\hline IA & 1.5 & 1 & $1.0-1.9$ & 1.2 & 0 & 4 \\
IBE & 2.5 & 2 & $2.0-3.0$ & 1.4 & 0 & 6 \\
IIAE & 6.5 & 7 & $5.4-7.6$ & 2.8 & 0 & 13 \\
IIBE & 6.6 & 6.5 & $5.4-7.7$ & 3 & 1 & 12 \\
IIIE & 6.0 & 6 & $4.7-7.3$ & 3.4 & 1 & 16 \\
IVE & 10.2 & 9.5 & $7.9-12.5$ & 5.9 & 2 & 27 \\
VE & 10.0 & 9.5 & $7.9-12.1$ & 5.5 & 0 & 25 \\
Total E & 43.3 & 41 & $39.4-47.2$ & 10.1 & 19 & 64 \\
\hline
\end{tabular}

CI. Confidence interval; SD. Standard deviation

When both sides of the neck were analyzed together, the mean number of LNs recovered from MRND specimen was 43.8 (range 19-99 LNs), with the highest mean of LNs in level V (11 LNs), compared to the other levels (Table 3).

TABLE 3 - Descriptive of both sides of the neck.

\begin{tabular}{ccccccc}
\hline N=56 & Mean & Median & CI $(95 \%)$ & SD & $\begin{array}{c}\text { Mini- } \\
\text { mum }\end{array}$ & $\begin{array}{c}\text { Maxi- } \\
\text { mum }\end{array}$ \\
\hline IA & 1.5 & 1 & $1.1-1.8$ & 1.2 & 0 & 4 \\
IB & 2.5 & 2 & $2.1-2.9$ & 1.4 & 0 & 8 \\
IIA & 7.2 & 6 & $6.0-8.5$ & 4.6 & 0 & 24 \\
IIB & 6.5 & 6 & $5.5-7.4$ & 3.6 & 0 & 15 \\
III & 6.6 & 7 & $5.7-7.4$ & 3.2 & 1 & 16 \\
IV & 8.6 & 8 & $7.1-10.1$ & 5.6 & 0 & 27 \\
V & 11 & 9.5 & $9.2-12.7$ & 6.5 & 0 & 34 \\
Total & 43.8 & 40.5 & $40.3-47.4$ & 13.2 & 19 & 99 \\
\hline
\end{tabular}

CI. Confidence interval; SD. Standard deviation

The comparative analysis of both sides of the neck (Table 4) showed no statistically significant difference, except for the number of LNs in level IV that was significantly higher in the left side $(7.0 \pm 4.9 \mathrm{LNs}$ in right side $v s .10 .2 \pm 5.9 \mathrm{LNs}$ in left side, $\mathrm{P}=0.026$ - Mann-Whitney's test).

TABLE 4 - Comparison of the number of lymph nodes recovered in each level in both sides of the neck.

\begin{tabular}{cccc}
\hline $\mathrm{N}=28$ & Right side & Left side & $\mathrm{p}^{*}$ \\
\hline IB & $2.5 \pm 1.5$ & $2.5 \pm 1.4$ & 0.825 \\
IIA & $8.0 \pm 5.8$ & $6.5 \pm 2.8$ & 0.987 \\
IIB & $6.3 \pm 4.2$ & $6.6 \pm 3.0$ & 0.494 \\
III & $7.1 \pm 2.9$ & $6.0 \pm 3.4$ & 0.142 \\
IV & $7.0 \pm 4.9$ & $10.2 \pm 5.9$ & 0.026 \\
V & $11.9 \pm 7.4$ & $10.0 \pm 5.5$ & 0.455 \\
Total & $44.4 \pm 16.0$ & $43.3 \pm 10.1$ & 0.560 \\
\hline
\end{tabular}

Mean \pm standard deviation (*Mann-Whitney's test)
The analysis of the nodal yield in relation to demographic and anthropometric data showed some statistically significant differences. Left side level IV had a higher mean number of LNs in males compared to females (respectively, 11.9 vs. $7.2 \mathrm{LNs} ; \mathrm{P}=0.040$ - Mann -Whitney test), and have shown positive correlation with height ( $r=+0.396, \mathrm{P}=0.037$ - Spearman's correlation test). Right side level $\mathrm{V}$ number of $\mathrm{LNs}$ had positive correlation with weight $(\mathrm{r}=+0.417, \mathrm{P}=0.027$ - Spearman's correlation test) and BMI $(\mathrm{r}=+0.456, \mathrm{P}=0.015$ - Spearman's correlation test $)$ of the subject. The total number of LNs at right side NDs showed positive correlation with age $(\mathrm{r}=+0.464, \mathrm{P}=0.013$ - Spearman's correlation test). There were no differences in relation to number of LNs and the race of individuals. Considering both neck sides, level V was positively correlated with weight $(\mathrm{r}=+0.278, \mathrm{P}=0.038$ - Spearman's correlation test) and $\mathrm{BMI}(\mathrm{r}=+0.317, \mathrm{P}=0.021$ - correlation test Spearman's) of the individuals and the total number of LNs in the specimen was negative correlated with age $(\mathrm{r}=-0.358, \mathrm{P}=0,007$ Spearman's correlation test), meaning that the number of LNs decreases with the increasing of age. Other associations did not present any significant difference (data not shown).

\section{Discussion}

This study identified the expected number of retrieved lymph nodes from each level of a modified radical neck dissection. There was also association of the nodal yield, in some specific situations, with sex, height, age, weight and body mass index of the individual. There are many studies accessing this issue, but showing a wide range in the mean number of lymph nodes identified in the specimens of MRND. Studies performed on cadaver model, which are not frequent in the literature, have shown lower nodal yields comparing to those found in this study. The confidence interval of the mean number of the LNs was calculated in order to improve external validity of the findings. To our knowledge, no similar approach has been published yet.

The nodal yield on MRND specimens identified at the present series was 43.8 (95\% CI: 40.3 - 47.4 LNs). Agrama et $a l^{3}$, in a review of the literature, analyzing 5228 neck dissections, found the mean of $35 \mathrm{LNs}$ in a RND specimen. Literature reports in clinical studies showed a mean of 31 to $57 \mathrm{LNs}^{6,9,13-17}$.

Only two studies published on cadaver model were found in the literature. Friedman et al. ${ }^{6}$, in an effort to provide reference for surgeons and pathologists regarding expected yield from selective ND, found the range of 6 to 44 in the number of LNs in specimens of RND in 20 cadavers. Norling et al..$^{18}$, as a result of the dissection of 9 cadavers, identified a range of 8 to 
24 LNs, but only six specimens contained the five nodal levels. Both studies were performed with a small sample and without the subdivision of levels I and II (IA and IB, IIA and IIB, respectively) recommended by the ND classification of the Committee for Head and Neck Surgery and Oncology the AAO- HNS ${ }^{11}$.

Compared to these previous studies in cadavers, we found a higher mean number of LNs retrieved from each nodal level, as well as in the whole specimen of the ND, with level V showing most significant difference. The nodal yield identified was also higher than those found in most studies with casuistics of patients undergoing ND as part of the treatment of HNSCC, except for the data from Kohler and Kowalski ${ }^{15}$ and Liao et al. ${ }^{16}$, who found a higher mean number. Although considering the possibility of hypertrophy and remodeling of cervical LNs, induced by the pathological state related to the presence of the primary tumor ${ }^{6,18}$, used by the authors of the cadaver studies to justify the lower number of LNs found, the present study reported the identification of a number of LNs comparable to those shown in clinical studies.

The results and their relationship to demographic and anthropometric data showed some statistically significant associations, but some correlation coefficients were low. The number of LNs in level IV, in the left side NDs, was higher in males (mean of 11.9 vs. 7.2 LNs for females) and had a positive correlation with height. This finding may be related to the position and anatomical variations of the omohyoid muscle ${ }^{19,20}$, which is considered the upper limit of level IV, while the clavicle is its lower limit. Accordingly, as the measure of the distance between this muscle and the clavicle is quite variable, it may influence on the amount of LNs recovered at this level ${ }^{19}$. However, studies directed to this anatomical region ${ }^{19,21}$ found no significant differences in measures of these distances related to sex, age and height.

The number of LNs on level V had positive correlation with weight and BMI of individuals, confirming findings of a previous study of lymph node content in patients undergoing ND diagnosed with melanoma ${ }^{22}$.

The total number of LN was negatively correlated with age, which was also described by Amat et al. ${ }^{17}$ and Norling et al. ${ }^{18}$, who attributed this relationship to the natural decrease in immune response and lymph node reactivity with aging.

Given the importance of ND in clinical and surgical practice of the treatment of HNSCC, and the wide variation of data available in the literature regarding the nodal yield, the main contribution of the present report was to calculate, with statistical accuracy, the mean number of LNs in the cervical nodal levels, as well as to estimate the confidence interval of this mean. Hence, a meaningful recommendation for a minimum number of LNs to be identified in the pathology specimen of a neck dissection could be established, suggesting a simple and practical quality control tool.

\section{Conclusion}

The results defined a parameter in relation to the minimum recommended nodal yield in a modified radical neck dissection, as well as the number of lymph nodes in each level of this dissection, performed in clinical practice.

\section{References}

1. Ferlito A, Rinaldo A, Robbins KT, Leemans CR, Shah JP, Shaha AR, Andersen PE, Kowalski LP, Pellitteri PK, Clayman GL, Rogers SN, Medina JE, Byers RM. Changing concepts in the surgical management of the cervical node metastasis. Oral Oncol. 2003;39(5):429-35. PMID: 12747966.

2. Jesse RH, Ballantyne AJ, Larson D. Radical or modified neck dissection: a therapeutic dilemma. Am J Surg. 1978;136(4):516-9. PMID: 707734.

3. Agrama MT, Reiter D, Topham AK, Keane WM. Node counts in neck dissection: are they useful in outcomes research? Otolaryngol Head Neck Surg. 2001;124(4):433-5. PMID: 11283502.

4. Bhattacharyya N. The effects of more conservative neck dissections and radiotherapy on nodal yields from the neck. Otolaryngol Head Neck Surg. 1998;124(4):412-6. PMID: 9559688.

5. Byers RM. Modified neck dissection. A study of 967 cases from 1970 to 1980. Am J Surg. 1985;150(4):414-21. PMID: 4051103.

6. Friedman M, Lim JW, Dickey W, Tanyeri H, Kirshenbaum GL, Phadke DM, Caldarelli D. Quantification of lymph nodes in selective neck dissection. Laryngoscope. 1999;109(3):368-70. PMID: 10089959.

7. Morton RP, Gray L, Tandon DA, Izzard M, McIvor NP. Efficacy of neck dissection: are surgical volumes important? Laryngoscope. 2009;119(6):1147-52. PMID: 19296506.

8. Seethala RR. Current state of neck dissection in the United States. Head Neck Pathol. 2009;3(3):238-45. PMID: 20596978.

9. van den Brekel MW, van der Waal I, Meijer CJ, Freeman JL, Castelijns JA, Snow GB. The incidence of micrometastases in neck dissection specimens obtained from elective neck dissections. Laryngoscope. 1996;106(8):987-91. PMID: 8699914.

10. Lwanga SK, Lemeshow S. Sample size determination in health studies: a practical manual. In: Organization WH, editor. Geneva; 1991.

11. Robbins KT, Clayman G, Levine PA, Medina J, Sessions R, Shaha A, Som P, Wolf GT; American Head and Neck Society; American Academy of Otolaryngology--Head and Neck Surgery. Neck dissection classification update: revisions proposed by the American Head and Neck Society and the American Academy of Otolaryngology-Head and Neck Surgery. Arch Otolaryngol Head Neck Surg. 2002;128(7):751-8. PMID: 12117328.

12. Robbins KT, Shaha AR, Medina JE, Califano JA, Wolf GT, Ferlito A, Som PM, Day TA; Committee for Neck Dissection Classification, American Head and Neck Society. Consensus statement on the classification and terminology of neck dissection. Arch Otolaryngol Head Neck Surg. 2008;134(5):536-8. PMID: 18490577.

13. Shah JP. Patterns of cervical lymph node metastasis from squamous carcinomas of the upper aerodigestive tract. Am J Surg. 1990;160(4):405-9. PMID: 2221244. 
14. Candela FC, Kothari K, Shah JP. Patterns of cervical node metastases from squamous carcinoma of the oropharynx and hypopharynx. Head Neck. 1990;12(3):197-203. PMID: 2358329.

15. Kohler HF, Kowalski LP. How many nodes are needed to stage a neck? A critical appraisal. Eur Arch Otorhinolaryngol. 2010;267(5):785-91. PMID: 19904547.

16. Liao CT, Hsueh C, Lee LY, Lin CY, Fan KH, Wang HM, Huang SF, Chen IH, Kang CJ, Ng SH, Tsao CK, Huang YC, Yen TC. Neck dissection field and lymph node density predict prognosis in patients with oral cavity cancer and pathological node metastases treated with adjuvant therapy. Oral Oncol. 2012;48(4):329-36. PMID: 22104249 .

17. Amar A, Chedid HM, Rapoport A, Cernea CR, Dedivitis RA, Curioni OA, Brandão LG. Prognostic significance of the number of lymph nodes in elective neck dissection for tongue and mouth floor cancers. Braz J Otorhinolaryngol. 2012;78(2):22-6. PMID: 22499366.

18. Norling R, Therkildsen MH, Bradley PJ, Nielsen MB, von Buchwald C. Nodal yield in selective neck dissection. Acta Otolaryngol. 2013;133(9):965-71. PMID: 23944948.

19. Mizen KD, Mitchell DA. Anatomical variability of omohyoid and its relevance in oropharyngeal cancer. Br J Oral Maxillofac Surg. 2005;43(4):285-8. PMID: 15993280.

20. Rai R, Ranade A, Nayak S, Vadgaonkar R, Mangala P, Krishnamurthy A. A study of anatomical variability of the omohyoid muscle and its clinical relevance. Clinics. 2008;63(4):521-4. PMID: 18719765.

21. Louzada AC, Lim SJ, Pallazzo JF, Silva VP, de Oliveira RV, Yoshio $\mathrm{AM}$, de Araújo-Neto $\mathrm{VJ}^{1}$, Leite $\mathrm{AK}^{1}$, Silveira $\mathrm{A}^{1}$, Simões $\mathrm{C}^{1}$, Brandão $\mathrm{LG}^{1}$, Matos $\mathrm{LL}^{2}$, Cernea $\mathrm{CR}$. Biometric measurements involving the terminal portion of the thoracic duct on left cervical level IV: an anatomic study. Anat Sci Int. 2016 Jun;91(3):274-9. PMID: 26272628

22. Rossi CR, Mozzillo N, Maurichi A, Pasquali S, Macripo G, Borgognoni L, Solari N, Piazzalunga D, Mascheroni L, Giudice G, Mocellin S, Patuzzo R, Caracò C, Ribero S, Marone U, Santinami $\mathrm{M}$. Number of excised lymph nodes as a quality assurance measure for lymphadenectomy in melanoma. JAMA Surg. 2014;149(7):7006. PMID: 24804856.

\section{Correspondence:}

Fábio de Aquino Capelli

Av. Dr. Enéas de Carvalho Aguiar, 255/8 andar, sala 8174

05403-000 São Paulo - SP Brasil

Tel.: (55 11)2661-6425

Fax: (55 11)2661-7506

fabiocapelli81@gmail.com

Received: Mar 23, 2016

Review: May 24, 2016

Accepted: Jun 23, 2016

Conflict of interest: none

Financial source: none

${ }^{1}$ Research performed at Department of Head and Neck Surgery, School of Medicine, Universidade de São Paulo (USP), Brazil. 